\title{
NÃO CONFORMIDADE DE GÊNERO E INFÂNCIA: REVISANDO A PRODUÇÃO CIENTÍFICA
}

\author{
GENDER NON-CONFORMITY AND CHILDHOOD: REVIEWING THE SCIENTIFIC PRODUCTION
}

\section{RESUMO}

Revisou-se sistematicamente a produção científica que abordasse a não conformidade de gênero na infância. Assim, pesquisaram-se os termos mais comuns nesta área "criança (e) transgênero" e "não conformidade de gênero (e) crianças" escritos em português, inglês e espanhol nas bases SciELO, BVS, Pepsic, PubMED, PsycINFO e CINAHL. Dos 836 artigos encontrados, incluíram-se 72 a partir de critérios de concordância. Analisaram-se os dados bibliométricos e de conteúdo através de três eixos com base no delineamento de pesquisa: (1) Aspectos gerais; (2) Cuidados com a saúde; (3) Questões relacionadas à família e (4) Temáticas diversas. Observa-se um crescimento na produção a partir de 2012 e uma concentração da publicação e dos pesquisadores nos EUA. Abordou-se principalmente o cuidado com a saúde através do uso de medicamentos e a dinâmica familiar. Sugerem-se pesquisas que abordem as especificidades no contexto brasileiro.

Palavras-chave:Não conformidade de gênero. Infância. Criança e revisão sistemática.

\begin{abstract}
We developed a systematic review of the scientific production that approached gender non-conforming in childhood. Thus, we searched the most common terms in this area "child (and) transgender" and "non-compliance of gender (and) children" written in Portuguese, English and Spanish in SciELO, VHL, Pepsic, PubMED, PsycINFO and CINAHL databases. Of the 836 articles found, 72 were included on the basis of criteria in concordance. Bibliometric and content data were analyzed through three axes based on the research design: (1) General aspects; (2) Health care; (3) Family related issues and (4) Miscellaneous topics. It is observed a growth in production from 2012 and a concentration of publication and researchers in the USA. The main focus was on health care through the use of medicines and family dynamics. We suggest researches to address the specificities of the Brazilian context.
\end{abstract}

Keywords: Gender non-conforming. Childhood. Children and systematic review.

Elder Cerqueira Santos,

Pós-Graduação em Psicologia, UFS. Email: eldercerqueira@gmail.com

Mariana Valadares de M Santana

UFS. Email: marianavms@hotmail.com 


\section{Introdução}

A infância se constitui como um período de desenvolvimento onde as regras sobre comportamento e papéis de gênero parecem ser mais rígidas do que na fase adulta. As cores das roupas, quais brinquedos usar ou quais esportes praticar, todas as possibilidades de experimentação da criança são regidas pela divisão do que pode um menino e do que pode uma menina (Bonassi, 2017). É na infância que as estilizações de gênero são internalizadas através de frases como: "homem não chora!" ou "sente-se como uma menina!" (Bento, 2006).

Não passa despercebido dos olhares dos seus cuidadores, quando uma criança expressa um desejo de brincar ou vestir-se de maneira pouco usual do que é determinado socialmente para o gênero que lhe foi designado. Dúvidas a respeito do que pode estar acontecendo com esta criança acabam por leva-las aos consultórios médicos e aos encontros com professores. Por que um menino quer brincar de boneca? Por que minha filha não gosta de ser tratada mais por pronomes femininos? A falta de informação sobre o que significa conceitos como expressão de gênero e identidade de gênero contribui para um sentimento de angústia frente à questão.

As expressões de gênero dizem respeito à maneira como a pessoa se apresenta, ou seja, sua aparência e seu comportamento, estas se relacionam com as expectativas sociais para um determinado gênero. Deste modo, os significados de ser mulher ou ser homem são múltiplos e podem variar de acordo com a cultura de cada lugar e tempo. Por exemplo, a moda que veste homens e mulheres e que representa essa divisão binária de cada período, pode ser compreendida como forma de expressão destes signos culturais de gênero a despeito dos costumes de cada sociedade. Assim, apesar de relacionadas, as expressões não são correspondentes absolutos da identidade de gênero da pessoa (Jesus, 2012).

A identidade de gênero seria um sentido interno, o gênero com o qual a pessoa se identifica, sendo que este pode ou não corresponder ao que foi designado no seu nascimento. Cisgênero é o termo utilizado para aquelas pessoas que se identificam com este gênero designado, enquanto Transgênero são aquelas que reivindicam outra auto-determinação qualquer (Bagagli, 2016; Dumaresq, 2014; Jesus, 2012).

Casos como o de Jazz Jennings tem ganhado cada vez mais notoriedade. Crianças e adolescentes que desde muito cedo põe em questão a identidade de gênero que lhe fora designada ao nascer, assim como a adolescente americana. Logo, pais e profissionais se percebem frente a questões de gênero que os desestabilizam e que demandam novas formas de cuidado. Diante da complexidade deste tema, volta-se à academia com questões como: de que maneira a produção científica dos últimos anos (2000-2016) tem estudado o tema? Quais as áreas de concentração? Quais as suas especificidades e que variáveis são estudadas? Deste modo, demandam-se revisões sistemáticas para que se possa compreender de que maneira a produção científica o tem pesquisado. 


\section{Método}

\section{Revisão Sistemática}

Este tipo de pesquisa caracteriza-se pela revisão crítica do material que já foi publicado através de um método estruturado que permita a sua reprodução (Bern, 1995). A sua aplicação justifica-se pela necessidade de compreender o panorama das publicações que abordem a temática, não somente ao descrever as características bibliométricas das pesquisas, mas ao desenvolver uma discussão que contribua para futuros trabalhos (Psychology Writing Center, 2005).

\section{Procedimentos}

A produção científica que abarca a temática utiliza uma gama variada de termos para se referir ao mesmo objeto. Ponderou-se que a escolha das palavras a serem utilizadas nesta revisão se daria pelo seu alcance nas pesquisas preliminares, ou seja, pela quantidade de artigos que apareciam como resposta. Sendo assim, optouse por "criança (e) transgênero", "transgender (e) children", "niños (e) transgénero", "crianças (e) não conformidade de gênero, "gender nonconforming (e) children" e "niños (e) no-conformidad de género" nas bases SciELO, BVS, Pepsic, PubMED, PsycINFO e CINAHL. Não houve limite de data inicial e foram incluídos artigos até abril de 2016.

Os critérios de inclusão foram: (1) abordar crianças que apresentassem expressões ou identidade de gênero compreendidas como dissidentes como sua temática; (2) ser um artigo científico; (3) ser escrito em português, inglês ou espanhol. Assim, encontraram-se 836 artigos, sem considerar as repetições. Destes, consideraram-se 72 como válidos neste trabalho. Quanto ao restante, excluiu-se com base nos seguintes critérios: (1) não abordou a temática transgênero em sua pesquisa, 25,4\%, (2) não abordou estas crianças como tema, 28,9\%; (3) caracterizou-se como um capítulo de livro, resenha de um livro, dissertação, tese, comentário, editorial, guia, 37,3\%; (4) não foi escrito em português, inglês ou espanhol, 3,5\%; (5) abordou somente o tema da categoria do DSM V, Disforia de Gênero, 2,7\%; (6) não se obteve acesso ao texto na íntegra, $2,1 \%$. 


\section{Análise de dados}

Bibliométrica

Categorizou-se e analisou-se estatisticamente parte dos dados utilizando o Statistical Package for the Social Sciences (SPSS V.23). Objetivou-se fornecer informações a respeito da origem dos artigos publicados e selecionados a fim de se construir um perfil amostral. Para isso, separou-se o país de origem do pesquisador, o país de origem da revista, as palavras-chave e área de formação do primeiro autor. Categorizou-se o método, o delineamento de pesquisa e os participantes.

\section{Conteúdo}

Na segunda fase do presente trabalho classificaram-se os artigos selecionados de acordo com o seu tema de pesquisa, a finalidade era produzir um quadro que permitisse vislumbrar os principais resultados e as diferentes abordagens metodológicas. Para isso, desenvolveram-se quatro eixos principais: (1) Aspectos gerais; (2) Cuidados com a saúde; (3) Questões relacionadas à família (4) Temáticas diversas.

\section{Resultados}

\section{Dados bibliométricos}

De um universo de 836 artigos pesquisados, foram considerados válidos apenas 72. Destacando-se o país de origem do primeiro autor, a maioria dos artigos inseridos na pesquisa foi produzida nos EUA (79,2\%), em seguida na Austrália (5,6\%), Canadá e Inglaterra (4,2\%), Espanha e Holanda, (2,8\%), e Brasil (1,4\%). Encontraram-se 53 revistas diferentes, sendo que as cinco com maior frequência são dos EUA: Journal of Homosexuality, 11,1\%; Family Process, 5,6\%; Pediatrics, International Journal of Transgenderism e Child Welfare, 4,2\%. A multiplicidade de termos utilizada para fazer referência ao mesmo objeto produziu uma gama de palavras-chave, sendo as quatro mais referidas: Transgênero, 36,1\%, Identidade de Gênero, 29,2\%, Variância de Gênero, 26,4\% e Disforia de Gênero, 16,7\%. Para clarificar a questão, separaramse os principais termos utilizados para referir-se a essa população, suas diferentes variações nas palavras-chave e suas frequências (Tabela 1). 
Tabela 1 - Palavras-chave

\begin{tabular}{|c|c|c|c|c|}
\hline Transgênero & $\begin{array}{l}\text { Variância de } \\
\text { Gênero }\end{array}$ & $\begin{array}{l}\text { Não conformidade } \\
\text { de Gênero }\end{array}$ & $\begin{array}{l}\text { Desordem de } \\
\text { Gênero }\end{array}$ & $\begin{array}{l}\text { Outras } \\
\text { palavras- } \\
\text { chave }\end{array}$ \\
\hline $\begin{array}{l}\text { Transgênero, } \\
36,1 \%\end{array}$ & $\begin{array}{l}\text { Variância de } \\
\text { Gênero, 26,4\% }\end{array}$ & $\begin{array}{l}\text { Não conformidade } \\
\text { de Gênero, 12,5\% }\end{array}$ & $\begin{array}{l}\text { Disforia de } \\
\text { Gênero, 16,7\% }\end{array}$ & $\begin{array}{l}\text { Discordância/ } \\
\text { Dissonância } \\
\text { de Gênero, } \\
\mathbf{1 , 4} \%\end{array}$ \\
\hline $\begin{array}{l}\text { Jovens } \\
\text { Transgênero, } \\
6,9 \%\end{array}$ & $\begin{array}{l}\text { Variância de } \\
\text { Gênero na } \\
\text { infância, 1,4\% }\end{array}$ & $\begin{array}{l}\text { Não conformidade } \\
\text { de Gênero na } \\
\text { infância, } 1,4 \%\end{array}$ & $\begin{array}{l}\text { Desordem(ns) } \\
\text { de Identidade } \\
\text { de Gênero na } \\
\text { infância, } 8,3 \%\end{array}$ & $\begin{array}{l}\text { Gênero } \\
\text { Atípico, 1,4\% }\end{array}$ \\
\hline $\begin{array}{l}\text { Criança } \\
\text { Transgênero, } \\
\text { 13,9\% }\end{array}$ & $\begin{array}{l}\text { Crianças } \\
\text { gênero } \\
\text { variantes, } \\
2,8 \%\end{array}$ & $\begin{array}{l}\text { Crianças em não } \\
\text { conformidade de } \\
\text { Gênero, } 8,3 \%\end{array}$ & $\begin{array}{l}\text { Desordem de } \\
\text { Identidade de } \\
\text { Gênero, } 13,9 \%\end{array}$ & $\begin{array}{l}\text { Desconforto } \\
\text { de Gênero, } \\
1,4 \%\end{array}$ \\
\hline
\end{tabular}

Em relação à área de formação do primeiro autor, percebe-se a predominância de três grandes áreas: Psicologia (43,1\%); Medicina (30,6\%); e Direito (6,9\%). Em relação ao ano de publicação, os artigos variavam entre o ano de 2000 e o de 2016. Os anos de 2000 e 2003 apresentaram 1,4\%, em 2006 foram 5,6\%, já em 2008 e 2009 foram $4,2 \%$, no ano de 2010 uma nova queda, $2,8 \%$, enquanto em 2011 foram $5,6 \%$. A partir do ano 2012 as publicações cresceram e se estabeleceram assim até o ano de 2016. Em 2012 e 2013 registraram-se 15,3\%, sendo os anos de 2014 e de 2015 com maior porcentagem, 16,7\%, enquanto até abril de 2016, 11,1\% (Figura 1).

Figura 1 - Número de Publicações

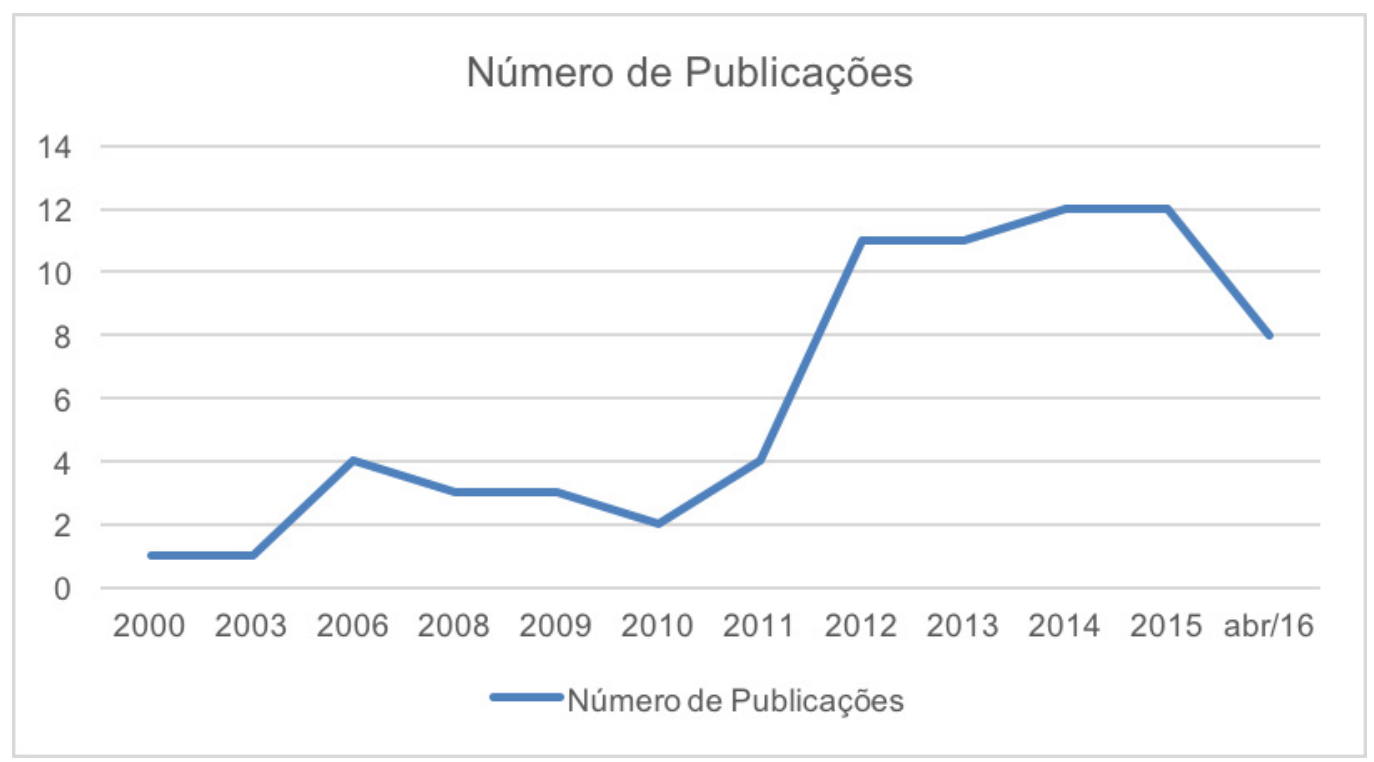


Entre os artigos inseridos prevaleceu-se o método qualitativo $(79,2 \%)$ em comparação ao quantitativo $(19,4 \%)$ e ao misto $(1,4 \%)$. Em relação ao delineamento de pesquisa, a maioria dos artigos utilizou o levantamento $(36,1 \%)$, seguido da revisão teórica $(23,6 \%)$, da pesquisa bibliográfica $(12,5 \%)$, do estudo de caso $(8,3 \%)$, ensaio teórico $(9,7 \%)$, da pesquisa documental e do relato de experiência $(4,2 \%)$ e, por fim, da pesquisa experimental $(1,4 \%)$. Nenhum tipo de instrumento padronizado foi manejado na maior parte dos trabalhos (63,9\%), e, quando se fez uso, os dois principais foram a entrevista semi-estruturada e o questionário autoral (11,1\%). Ainda surgiram casos de questionário replicado $(8,3 \%)$, entrevista não-estruturada $(2,8 \%)$, escalas e entrevista estruturada $(1,4 \%)$.

Identificaram-se quem são os sujeitos abordados diretamente nas pesquisas. As crianças trans representaram $18,1 \%$, enquanto seus pais foram ouvidos por $13,9 \%$. Adultos trans e adultos em geral participaram de 8,3\% dos trabalhos, profissionais de saúde $6,9 \%$, enquanto professores e crianças em geral somente 1,4\%. Em sua maioria, as pesquisas não tiveram a participação de nenhum tipo de sujeito, 54,2\%.

\section{Resultados}

\section{Conteúdo}

Eixo 1: Aspectos gerais

Este eixo engloba pesquisas que apresentaram um panorama sobre a questão e, assim, que objetivaram construir certo conhecimento básico que contribuísse para futuras investigações. No caso dos artigos aqui selecionados, notou-se um uso recorrente da descrição de terminologias que abrangessem a temática sem que, contudo, as suas definições estivessem completamente explícitas ou ainda correspondessem necessariamente a um consenso (Tabela 2).

Tabela 2 - Aspectos gerais

Eixo 1: Aspectos gerais

\begin{tabular}{l|l}
\hline Pesquisas bibliográficas e documentais & $\begin{array}{l}\text { Aramburu (2016); Kelso (2015); Ryan \& } \\
\text { Rivers (2003); Scherrer \& Woodford (2013) }\end{array}$ \\
\hline Revisões teóricas & $\begin{array}{l}\text { Bonifacio \& Rosenthal, 2015; Simons, } \\
\text { Leibowitz \& Hidalgo, 2014; Leibowitz \& } \\
\text { Telingator, 2012; \& Pleak, 2011 }\end{array}$ \\
\hline
\end{tabular}


A saúde é um tema amplo de pesquisa e, por isso, tomou-se como critério de inclusão para este eixo os artigos que tivessem como base de discussão os cuidados relativos ao atendimento profissional às crianças que chegam aos consultórios e aos ambulatórios e, em um caso, à escola (Tabela 3).

Tabela 3 - Cuidados com a saúde

Eixo 2: Cuidados com a saúde

\begin{tabular}{|c|c|}
\hline Pesquisas bibliográficas e documentais & $\begin{array}{l}\text { Castañeda (2015); Fernández Rodríguez, } \\
\text { Guerra Mora \& García-Veja (2014); } \\
\text { Vance, Ehrensaft \& Rosenthal (2014); } \\
\text { Adelson (2012); Stieglitz (2010); Mallon \& } \\
\text { DeCrescenzo (2006) }\end{array}$ \\
\hline Revisões teóricas & $\begin{array}{l}\text { Drescher \& Pula (2014); Wren (2014); } \\
\text { Forcier \& Haddad (2013); Wallace \& } \\
\text { Russell (2013); Bernal \& Coolhart (2012); } \\
\text { Schwartz (2012); Stein (2012); Ehrensaft } \\
\text { (2011); Stoddard, Leibowitz, Ton, Snowdon } \\
\text { (2011); de Vries, \& Cohen-Kettenis (2009); } \\
\text { Menvielle (2009); Vanderburgh(2009); } \\
\text { Mallon \& Woronoff (2006); Halderman } \\
\text { (2000) }\end{array}$ \\
\hline
\end{tabular}

Alessi, Kahn \& Chatterji (2016); Drescher \& Byne (2012); Ehrensaft (2014); Mahdi, Jevertson, Schrader, Nelson, \& Ramos (2014); Olson, Durwood, DeMeules \& McLaughlin (2016); Pauletti, Cooper \& Perry (2014); Payne \& Smith (2014); Platero (2014); Rieger, Linsenmeier, Gygax, \& Bailey (2008); Riley, Clemson, Sitharthan \& Diamond (2013); Roberts,

Levantamentos Rosario, Corliss, Koenen, \& Austin (2012); Saketopoulou (2014); Shumer, Roberts, Reisner, Sari, Lyall \& Austin (2015); Silva \& Oliveira (2015); Slesaransky-Poe, Ruzzi, Dimedio, \& Stanley (2013); Steensma, van der Ende, Verhulst, \& Cohen-Kettenis (2013); Tishelman, Kaufman, EdwardsLeeper, Mandel, Shumer \& Spack (2015); Vance, Halpern-Felsher \& Rosenthal (2015); Wiseman \& Davidson (2012) 
No âmbito da clínica, a principal discussão é sobre o tratamento que se confere nestes casos. Reclamam-se não só as teorizações sobre gênero e identidade de gênero, mas a eficácia de cada metodologia construída a partir delas. Como exemplos, apresentam-se os trabalhos sobre o massive gender trauma de Saketopoulou (2014), no qual se busca construir uma relação entre a criança e seu corpo que não seja de sofrimento, e o true gender self de Ehrensaft (2014), que tem como objetivo estabelecer uma relação entre a criança e seu meio, ao mesmo tempo em que esta busca seu autêntico self de gênero.

Contudo, há uma noção que permeia a maioria das reverberações sobre as ações terapêuticas, sendo esta a importância de se trabalhar em uma equipe multidisciplinar composta por profissionais da área da saúde, saúde mental e assistência social (Mahdi et al., 2014; Payne \& Smith, 2014; Platero, 2014). Argumentase que as particularidades provenientes da situação demandam diferentes suportes e acolhimentos, ainda que a medicina seja comumente a porta de entrada num serviço de saúde. Todavia, os dados também apontam que em um primeiro momento, tanto os profissionais de saúde quanto os escolares sentem certa ansiedade por conta destas mesmas especificidades, a exemplo de uma dificuldade em reformular as normas de gênero que permeiam tradicionalmente seus trabalhos.

Salienta-se a preocupação dos autores com eventos estressores vivenciados por essas crianças e que apresentam correlação com posteriores casos de depressão e ansiedade, contexto também inserido em muitas realidades de jovens LGBT (Hatzenbuehler, 2011). Neste sentido, as pesquisas sobre auto-percepção e desenvolvimento cognitivo demonstram que um ambiente acolhedor favorece o crescimento saudável em termos de saúde mental e que, quando criadas sob a identidade de gênero que se reconhecem, as crianças demonstram o mesmo parâmetro de desenvolvimento cognitivo que crianças que não demandam atenção para questões de gênero (Olson et al., 2016).

Duas pesquisas se dedicaram a estudar vinculações com outros quadros de saúde, como o Autismo e a Disforia de Gênero. Shumer et al. (2015) formam um grupo de enfermeiras que encontrou correlações significativas entre traços de autismo nas crianças e em suas mães e a não conformidade de gênero. Já Drescher \& Byne (2012) realizaram um levantamento entre os principais autores, a fim de reunirem informações sobre a Disforia de Gênero e a variância de gênero. Entretanto, vale ressaltar, que os mesmos usaram os dois termos como sinônimos. Neste mesmo sentido, pesquisas buscaram correlações significativas entre não conformidade de gênero na infância com orientação sexual na vida adulta, obtendo uma ligação entre a homossexualidade e não conformidade na infância (Steensma et al., 2013; Rieger et al., 2008). 
Eixo 3 - Questões relacionadas à família

A terceira temática mais recorrente foi questões que envolvessem a família. Isto quer dizer sobre suporte parental a estas crianças e qual papel as questões de gênero têm desempenhado na dinâmica familiar.

Tabela 4 - Questões relacionadas à família

Eixo 3: Questões relacionadas à família

\begin{tabular}{l|l}
\hline Pesquisa bibliográfica & $\begin{array}{l}\text { Dierckx, Motmans, Mortelmans \& T'sjoen } \\
(2015)\end{array}$ \\
\hline \multirow{2}{*}{ Levantamentos } & $\begin{array}{l}\text { Gray, Sweeney, Randazzo \& Levitt (2016); } \\
\text { Gonzalez, Rostosky, Odom \& Riggle } \\
(2013) ; \text { Istar Lev (2010); Kuvalanka, Weiner } \\
\& \text { Mahan (2014); Malpas (2011); Pyne } \\
(2016) ; \text { Rahilly (2015); Tyler (2015); Riley, } \\
\text { Sitharthan, Clemson, \& Diamond (2013). }\end{array}$ \\
\hline
\end{tabular}

As pesquisas buscaram compreender a relação entre os pais e os seus filhos, bem como o processo de transição social e qual a rede de sentidos e significados dessa experiência (Gray et al., 2016; Pyne, 2016; Tyler, 2015; Gonzalez et al., 2013; Malpas, 2011; Lev, 2010). Pesquisas que trabalham com a memória das pessoas trans adultas destacam a importância do suporte parental durante as fases da infância e juventude no desenvolvimento destas pessoas (Kich, 2011; Jesus, 2013; Silva \& Oliveira, 2015).

A fim de estabelecer este suporte para seus filhos, as pesquisas apontaram que os pais desenvolvem uma consciência crítica frente às normas de gênero e buscam novas estratégias para educá-los (Rahilly, 2015). São observados os comportamentos e as atitudes das crianças que fazem uma transição de gênero, assim como se ressalta a necessidade do envolvimento com a comunidade. Tanto os profissionais da escola, quanto os de saúde que lidam com a criança ajudam e são ajudados por esses pais para que possam também ampliar o seu repertório de trabalho e consigam promover acolhimento a esta família (Kuvalanka et al., 2014; Riley et al., 2013).

\section{Eixo 4: Temáticas Diversas}

Por fim, o Eixo 4 reúne pesquisas que trabalharam esta população, mas que abordaram temáticas diversas, como: direitos civis, religião, opinião de adultos sobre estas crianças, abuso sexual e agressão, além da opinião de profissionais de saúde e professores. 
Tabela 5 - Temáticas diversas

Eixo 4: Temáticas diversas

\begin{tabular}{|c|c|c|}
\hline \multirow[t]{2}{*}{ Revisões teóricas } & Revisões sobre direitos civis & $\begin{array}{l}\text { Estrada \& Marksamer (2006); } \\
\text { Holman \& Goldberg (2006); Jeffreys } \\
\text { (2012); Meyer \& Bayer (2013). }\end{array}$ \\
\hline & Revisão sobre religião & Rubano (2015). \\
\hline \multirow{3}{*}{ Levantamentos } & Levantamento sobre religião & $\begin{array}{l}\text { García, Gray-Stanley \& Ramirez- } \\
\text { Valles (2008). }\end{array}$ \\
\hline & $\begin{array}{l}\text { Levantamentos sobre a } \\
\text { visão de adultos a respeito } \\
\text { de crianças em não } \\
\text { conformidade de gênero }\end{array}$ & $\begin{array}{l}\text { Coyle, Fulcher, \& Trübutschek (2016); } \\
\text { Elischberger, Glazier, Hill, Eric \& } \\
\text { Verduzco-Baker (2016); Thomas, \& } \\
\text { Blakemore (2013) }\end{array}$ \\
\hline & $\begin{array}{l}\text { Levantamentos sobre abuso } \\
\text { sexual e agressão }\end{array}$ & $\begin{array}{l}\text { Alessi, Kahn \& Chatterji (2016); } \\
\text { Pauletti, Cooper \& Perry (2014); } \\
\text { Roberts, Rosario, Corliss, Koenen, } \\
\text { \& Austin (2012) }\end{array}$ \\
\hline
\end{tabular}

Como visto anteriormente, ainda que a relação entre os pais e seus filhos esteja implicada com o grau de informação de cada família e com a comunidade em que está inserida, de maneira geral, os pais estão mais preocupados com o preconceito e os estigmas aos quais seus filhos estão expostos. Estas apreensões dos pais quanto à sociedade mostram-se válidas, pois a desinformação dos adultos acerca da temática aparece correlacionada a um viés de estigma, o qual desencadeia posicionamentos excludentes, como a não aceitação do uso de banheiros ou quartos compartilhados com outras crianças. (Elischberger et al., 2016; Coyle et al., 2016). Este tipo de violência agrava-se quando associada ao abuso sexual, violência física e psicológica, as quais essa população aparece como mais suscetível a ser vítima, como apontam as pesquisas (Alessi et al., 2016; Pauletti et al., 2014; Roberts et al., 2012). Neste mesmo sentido, a religião católica aparece no estudo de García et al. (2008) como um elemento que afasta a comunidade LBGT, mesmo que durante a infância tenha sido participado frequentemente da igreja. Assim, os pais assumem muitas vezes uma postura de militância por uma sociedade mais inclusiva e advogam pela luta dos direitos civis da população trans.

Estas dificuldades aos quais a família está exposta parece estar relacionada também a falta de parâmetros legais para lidar com certas situações. Os artigos que abordam este tema são dos EUA e da Austrália, países em que o debate ocorre em escala nacional. Buscam-se estabelecer protocolos de atendimento a esta população dentro de escritórios legais, o limite entre liberdade de expressão religiosa e ações 
afirmativas em escolas, assim como se debate como o Estado deve lidar com as crianças e os jovens que estão sob sua custódia.

\section{Discussão}

A revisão destes artigos apresenta uma primeira problemática para a discussão dos seus resultados: estes autores estão discutindo o mesmo objeto de estudo? A variabilidade de termos aponta para uma compreensão igualmente diversa do tema. Em certos autores, os quais trabalham com termos como "variância de gênero", aborda-se tanto crianças que demandam questões de identidade de gênero, quanto aquelas que expressam comportamentos compreendidos como atípicos para o seu sexo biológico. Ainda há autores que interpretam todas estas questões como um sintoma para a classificação da Disforia de Gênero na infância, a qual corresponde a uma classificação nosológica. Portanto, salienta-se a importância de se estabelecer qual a área de concentração da pesquisa e do seu autor, assim como o termo utilizado para descrever o objeto de estudo.

Esta questão parece estar relacionada com o fato de que muito recentemente ocorreu o aumento do número de pesquisas que abordam o tema como apontaram os dados bibliométricos. O perfil amostral construído corrobora a hipótese de que o campo da não conformidade de gênero na infância tem impacto recente na academia. Justifica-se pelo aumento das produções a partir do ano de 2012, sendo que há uma grande probabilidade do ano de 2016 apresentar um novo crescimento significativo. Salienta-se também a escolha por revistas com alto fator de impacto, por exemplo a Pediatrics possuía 5.473 de fator de impacto em 2014. Esse recente interesse é justificado por diversos autores devido ao crescimento do atendimento dessas crianças nos consultórios de saúde (Garofalo, 2014; Drescher \& Pula, 2014; Olson et al., 2016).

Ainda há uma problemática da ausência das questões de gênero nas grades curriculares dos profissionais, que chegam ao campo sem um suporte teórico em sua formação. Assim, devido a esta demanda precisam se dedicar aos estudos das questões de gênero quando já estão na prática, o que resulta em uma divergência muito grande sobre as compreensões em relação ao cuidado destas crianças. A variabilidade de termos encontrada nesta revisão corrobora esta hipótese. Assim, as famílias são expostas e sobrecarregadas por uma gama de profissionais e opiniões a respeito de suas crianças e sentem uma grande angústia frente à decisão de que cuidados ter para com as mesmas (Drescher \& Byne, 2012). Por isso, foi interessante notar que as pesquisas reforçaram o trabalho de uma equipe multidisciplinar para os cuidados com a saúde desta criança. Seja para que os profissionais estejam atentos aos diversos aspectos da sua saúde, seja para que possam trocar informações e criar estratégias que tenham resultados mais satisfatórios na produção de seu bem estar e desenvolvimento saudável. 
De acordo com a WPATH (2012), o cuidado despendido a este público é primordialmente acolhedor, buscando-se levar em consideração as singularidades de cada sujeito, sua família e sua história. O argumento da WPATH é de que se construa um ambiente saudável para o desenvolvimento desta criança e para suas experimentações a respeito da expressão de gênero. Ainda que se afirme a existência de um componente ontogenético a respeitos das representações sociais e da identidade de gênero em diferentes culturas, é preciso que a postura do profissional seja de acolhimento do processo singular de cada criança (Carli \& Bukatko, 20oo; Duveen, 1993). Esta criança que se expressa de maneira não conforme pode ser colocada num lugar da diferença não só pelos adultos, mas também por outras crianças. Mesmo que os adultos mais próximos das crianças não reproduzam uma estereotipia de gênero, elementos da macrocultura são assimilados por elas, interpretados e repetidos na sua microssociedade (Carvalho \& Pedrosa, 2002; Cerqueira-Santos \& Bourne, 2015). O espaço da brincadeira que é onde ocorrem as experimentações de gênero é também aquele em que essas regras se estabelecem e onde os deslocamentos ressaltam.

Destaca-se que qualquer tipo de intervenção medicamentosa só pode ser administrada a partir da entrada da criança na puberdade, na qual há uma suspensão do desenvolvimento das características sexuais secundárias até que o jovem tenha idade suficiente para que se prescrevam os hormônios. Este acolhimento diz respeito também a esta família e a esta comunidade que a partir dos comportamentos e sentimentos desta criança é posta diante das questões de gênero (Preciado, 2013). Além disso, diferente dos adultos, o menor de idade não tem como decidir legalmente se irá passar por algum tratamento e qual seria este tratamento.

Os artigos aqui selecionados tratavam sobre pais que buscaram auxílio para as questões de gênero demandadas por suas crianças, esta simples atitude ocasiona um recorte diante da realidade. As pesquisas apontam que, de maneira geral, a família é o primeiro espaço para algum tipo de violência homofóbica contra pessoas LGBTs (Perucchi et al., 2014). A despeito deste viés, os resultados encontrados estão em consonância com o argumento da importância de uma rede social de apoio para a autoestima destas pessoas (Silva \& Cerqueira-Santos, 2014). Destaca-se a atitude de militância assumida pelos pais e responsáveis que buscam não só legitimar os direitos de seus filhos, mas também implica na discussão da temática de modo mais amplo na sociedade, afim de que se possa reduzir o viés de estigma social associado a estas experiências de gênero.

\section{Conclusão}

Diantedosobjetivosapresentados, arevisãosistemáticadeliteraturaapresentouse como o melhor método para delimitar o perfil amostral, tanto bibliométrico, como por conteúdo dos resultados. Entretanto, é evidente a dificuldade de se resumir os resultados encontrados nas pesquisas, devido às diferentes perspectivas a respeito 
deste objeto de estudo. Enquanto os pais ou cuidadores encontram-se cheios de dúvidas e anseios a respeito do futuro da criança, os profissionais buscam suprir a falta de base teórica de suas formações através das mais variadas formulações.

Compreende-se, então, porque o cuidado com a saúde e as questões relacionadas à família foram as temáticas mais abordadas. Apesar das diferentes abordagens, convergem-se os argumentos sobre a necessidade de um apoio social para esta família, bem como da construção de um ambiente saudável para estas crianças. A atitude do profissional da saúde ou da educação deve ser permeada por uma postura ética de respeito à singularidade do indivíduo e da maneira como ocorre este processo de desenvolvimento. Esta temática delicada envolve a frustração dos pais a respeito do filho imaginado diante do filho real, assim como de seus medos e angústias frente ao outro que poderá assumir uma atitude de segregação.

Ressalta-se a importância da continuidade de pesquisas na área, bem como do desenvolvimento de ferramentas que auxiliem a criança, a família e a comunidade em que está inserida.

\section{Referências}

ADELSON, S. L. 2012. Practice Parameter on Gay, Lesbian, or Bisexual Sexual Orientation, Gender Nonconformity, and Gender Discordance in Children and Adolescents. J Am Acad Child Adolesc Psychiatry. V. 9, №5, P. 957-974.

ALESSI, E.J., KAHN, S. e CHATTERJI, S. 2016. "The darkest times of my life": Recollections of child abuse among forced migrants persecuted because of their sexual orientation and gender identity. Child abuse and neglect, V. 51, p. 93-105.

AMERICAN ACADEMY OF CHILD \& ADOLESCENT PSYCHIATRY (2012). Practice parameter on gay, lesbian, or bisexual sexual orientation, gender nonconformity, and gender discordance in children and adolescents. Journal of the American Academy of Child \& Adolescent Psychiatry, V. 51, no 9, p. 957-974.

ARAMBURU, A. C. 2016. Gender nonconforming and transgender children/youth: Family, community, and implications for practice. Journal of the American Association of Nurse practitioners.

BAGAGLI, B. P. 2016. A diferença trans no gênero para além da patologização. Periódicus, V. 1, no. 5, p. 87-100.

BERN, D. J. 1995. Writing a review article for Psychological Bulletin. Psychological Bulletin, V. 118, no 2, p. 172-177. 
BERNAL, A. T. e COOLHART, D. 2012. Treatment and ethical considerations with transgender children and youth in family therapy. Journal of Family Psychotherapy, V. 23, no 4, p. 287-303.

BENTO, B. 2006. A reinvenção do corpo: sexualidade e gênero na experiência transexual. Rio de Janeiro, RJ: Garamond

CARLI, L.L.; BUKATKO, D. 200o. Gender, communication, and social influence. A developmental perspective. In:T. ECKES; H.M. TRAUTNER (eds.), The developmental social psychology of gender. Mahwah, Lawrence Erlbaum Associates, p. 295-331.

CARVALHO, A. M. A. e PEDROSA, M. I. 2002. Cultura no grupo de brinquedo. Estudos de Psicologia, V. 7, no 1, p. 181-188.

CASTAÑEDA, C. 2015. Developing gender: The medical treatment of transgender young people. Social science and medicine. V. 143, p. 262-70.

CERQUEIRA-SANTOS, E., BOURNE, J. 2016. Estereotipia de Gênero nas Brincadeiras de Faz de Conta de Crianças Adotadas por Casais Homoparentais. Psico-USF, V. 21, no 1, p. 125-133.

COYLE, E. F., FULCHER, M. e TRÜBUTSCHEK, D. 2016. Sissies, mama's boys, and tomboys: Is children's gender nonconformity more acceptable when nonconforming traits are positive? Archives of Sexual Behavior.

DE VRIES, A. L. C. e COHEN-KETTENIS, P. T. 2009. Review of World Professional Association for Transgender Health's Standards of Care for children and adolescents with gender identity disorder: A need for change? International Journal of Transgenderism, V. 11, no 2, p. 100-109.

DIERCKX, M., MOTMANS, J., MORTELMANS, D. e T'SJOEN, G. 2016. Families in transition: A literature review. International review of psychiatry, V. 28, no 1, p. 36-43.

DUMARESQ, L. 2016. Ensaio (travesti) sobre a escuta (cisgênera). Periódicus. V. 1, no 5, p. 121-131.

DUVEEN, G. 1993. The development of social representation of gender. Papers on Social Representations, 2(1):171-177.

DRESCHER, J. e BYNE, W. 2012. Gender dysphoric/gender variant (GD/GV) children and adolescents: Summarizing what we know and what we have yet to learn. Journal of Homosexuality, V. 59, no 3, p. 501-510. 
DRESCHER, J. e PULA, J. 2014. Ethical issues raised by the treatment of gender-variant prepubescent children. Hastings Center report, V. 44, p. 17-22.

EHRENSAFT, D. 2011. Boys will be girls, girls will be boys: Children affect parents as parents affect children in gender nonconformity. Psychoanalytic Psychology, V. 28, no 4, p. 528-548.

EHRENSAFT, D. 2012. From gender identity disorder to gender identity creativity: true gender self child therapy. Journal of Homosexuality, V. 59, № 3, p. 337-56.

EHRENSAFT, D. 2014. Found in transition: Our littlest transgender people. Contemporary Psychoanalysis, V. 50, nº 4, p. 571-592.

ELISCHBERGER, H. B., GLAZIER, J. J., HILL, E. D. e VERDUZCO-BAKER, L. 2016. "Boys don't cry"-or do they? Adult attitudes toward and beliefs about transgender youth. Sex Roles.

ESTRADA, R. e MARKSAMER, J. 2006. The legal rights of LGBT youth in state custody: what child welfare and juvenile justice professionals need to know. Child Welfare, V. 85 , no 2, p. 171-94.

FERNÁNDEZ RODRÍGUEZ, M., GUERRA MORA, M. e GARCÍA-VEGA, E. 2014. La $7^{\text {a }}$ versión de los Estándares Asistenciales de la WPATH. Un enfoque diferente que supera el dimorfismo sexual y de género. Revista de la Asociación Española de Neuropsiquiatría, V. 34, no 122, p. 317-335.

FORCIER, M. M. e HADDAD, E. 2013. Health care for gender variant or gender nonconforming children. Rhode Island medical journal, V. 96, no 4, p. 17-21.

GARCÍA, D. I., GRAY-STANLEY, J. e RAMIREZ-VALLES, J. 20o8. “The priest obviously doesn't know that I'm gay": the religious and spiritual journeys of Latino gay men. Journal of Homosexuality, V. 55, no 3, p. 411-36.

GAROFALO, R. 2014. Tipping Points in Caring for the Gender-Nonconforming Child and Adolescent. Pediatric Annals, V. 43, p. 227-229.

GONZALEZ, K. A., ROSTOSKY, S. S., ODOM, R. D. e RIGGLE, E. D. 2013. The positive aspects of being the parent of an LGBTQ Child. Family Process, V. 52, no 2, p. 325-37.

GRAY, S. A., SWEENEY, K. K., RANDAZZO, R. e LEVITT, H. M. 2016. “Am I Doing the Right Thing?": Pathways to Parenting a Gender Variant Child. Family Process, V. 55, no 1 , p. 123-38. 
HALDERMAN, D. C. 20oo. Gender atypical youth: Clinical and social issues. School Psychology Review, V. 29, no 2, p. 192-200.

HATZENBUEHLER, M. L. 2011. The Social Environment and Suicide Attempts in Lesbian, Gay, and Bisexual Youth. Pedriatrics, V. 127, no 5, p. 896-905.

HOLMAN, C. W. e GOLDBERG, J. M. 2006. Social and medical transgender case advocacy. International Journal of Transgenderism, V. 9, no 3/4, p. 197-217.

INSTITUTE OF MEDICINE. 2011. The health of lesbian, gay, bisexual, and transgender people: Building a foundation for better understanding. Washington, DC: The National Academies Press.

ISTAR LEV, A. 2010. How queer!--the development of gender identity and sexual orientation in LGBTQ-headed families. Family Process, V. 49, no 3, p. 268-90.

JEFFREYS, S. 2012. The transgendering of children: Gender eugenics. Women's Studies International Forum, V. 35, no 5, p. 384-393.

JESUS, J. G. 2012. Orientações sobre identidade de gênero: conceitos e termos. Brasília

JESUS, J. G. 2013. Crianças trans: memórias e desafios teóricos. In III Seminário Internacional Enlaçando Sexualidades. Salvador, Bahia: Universidade do Estado da Bahia

KELSO, T. 2015. Still Trapped in the U.S. Media's Closet: Representations of GenderVariant, Pre-Adolescent Children. Journal of Homosexuality, V. 62, no 8.

KICH, F. D. Singularidades em narrativas de transexuais: entre a performance e a norma. Dissertação de mestrado, Universidade Federal de Sergipe, Brasil, 2011.

KUVALANKA, K. A., WEINER, J. L. e MAHAN, D. 2014. Child, family, and community transformations: Findings from interviews with mothers of transgender girls. V. 10, oㅡ 1-2, p. 145-167.

LEIBOWITZ, S. F. e TELINGATOR, C. 2012. Assessing Gender Identity Concerns in Children and Adolescents: Evaluation, Treatments, and Outcomes. Current Psychiatry Reports, V. 14, p. 111-120.

MAHDI, I., JEVERTSON, J., SCHRADER, R., NELSON, A. e RAMOS, M. M. 2014. Survey of New Mexico School Health Professionals Regarding Preparedness to Support Sexual Minority Students. Journal of School Health, V. 84, no 1, p. 18-24. 
MALLON, G. P. e DECRESCENZO, T. 2006. Transgender children and youth: a child welfare practice perspective. Child Welfare, V. 85, no 2, p. 215-41.

MALLON, G. P. e WORONOFF, R. 2006. Busting out of the child welfare closet: lesbian, gay, bisexual, and transgender-affirming approaches to child welfare. Child Welfare, V. 85, no 2, p.115-22.

MALPAS, J. 2011. Between pink and blue: A multi-dimensional family approach to gender nonconforming children and their families. Family Process, V. 5o, no 4, p. 453470.

MENVIELLE, E. 2009. Transgender children: Clinical and ethical issues in prepubertal presentations. Journal of Gay E Lesbian Mental Health, V. 13, no 4, p. 292-297.

MEYER, I. H. e BAYER, R. 2013. School-Based Gay-Affirmative Interventions: First Amendment and Ethical Concerns. American Journal of Public Health, V. 103, no 10, p. 1764-1771.

MINTER, S. P. 2012. Supporting Transgender Children: New Legal, Social, and Medical Approaches. Journal of Homosexuality, V. 59, p. 422-433.

MORGAN, S. W. e STEVENS, P. E. 2008. Transgender identity development as represented by a group of female-to-male transgendered adults. Issues in Mental Health Nursing, V. 29, no 6, p. 585-99.

OLSON, K. R. 2016. Prepubescent transgender children: what we do and do not know. Journal of the American Academy of Child E Adolescent Psychiatry, V. 55, no 3, p. 155156.

OLSON, K. R., KEY, A. C. e EATON, N. R. 2015. Gender cognition in transgender children. Psychological science, V. 26, no 4, p. 467-74.

OLSON, K. R., DURWOOD, L., DEMEULES, M. e MCLAUGHLIN, K. A. (2016). Mental Health of Transgender Children Who Are Supported in Their Identities. Pediatrics, V. 137, no 3 , p. 1-8.

PAULETTI, R. E., COOPER, P. J. e PERRY, D. G. 2014. Influences of gender identity on children's maltreatment of gender-nonconforming peers: a person $\times$ target analysis of aggression. Journal of Personality and Social Psychology, V. 106, no 5, p. 843-66.

PAYNE, E. e SMITH, M. 2014. The big freak out: educator fear in response to the presence of transgender elementary school students. Journal of Homosexuality, V. 61, no 3 , p. 399-418. 
PERUCCHI, J., BRANDÃO, B. C., \& VIEIRA, H. I. dos S. 2014. Aspectos psicossociais da homofobia intrafamiliar e saúde de jovens lésbicas e gays. Estudos de Psicologia (Natal), V.19, no (1), 67-76. https://dx.doi.org/10.1590/S1413-294X2014000100009

PLATERO, R. 2014. The influence of psychiatric and legal discourses on parents of gender-nonconforming children and trans youths in Spain. Journal of GLBT Family Studies, V. 10, no 1-2, p. 145-167.

PLEAK, R. R. 2011. Gender identity issues in youth: opportunities, terminologies, histories, and advancements. Child and adolescent psychiatric clinics of North, Child \& Adolescent Psychiatric Clinics of North America, V. 20, no 4, p. 601-25.

PRECIADO, P. B. 2013. Quem defende a criança queer? Revista Geni, V.16.

PYNE, J. 2016. "Parenting is not a job... It's a relationship": Recognition and relational knowledge among parents of gender non-conforming children. Journal of Progressive Human Services, V. 27, no 1, p. 21-48

RAHILLY, E. P. 2015. The Gender Binary Meets the Gender-Variant Child: Parents' Negotiations with Childhood Gender Variance. Gender E Society, V. 20, no 10, p. 1-24.

RIEGER, G., LINSENMEIER, J. A. W., GYGAX, L. eBAILEY, J. M. 20o8. Sexual orientation and childhood gender nonconformity: Evidence from home videos. Developmental Psychology, V. 44, no 1, p. 46-58.

RIJN, A. B., STEENSMA, T. D., KREUKELS B. P. e COHEN-KETTENIS, P. T. 2013. Selfperception in a clinical sample of gender variant children. Clinical Child Psychology and Psychiatry, V. 18, no 3 , p. 464-74.

RILEY, E. A., CLEMSON, L., SITHARTHAN, G. e DIAMOND, M. 2013. Surviving a gender-variant childhood: the views of transgender adults on the needs of gendervariant children and their parents. Journal of sex and marital therapy, V. 39, no 3, p. 241-63.

RILEY, E. A, SITHARTHAN, G., CLEMSON, L. e DIAMOND, M. 2013. Recognising the needs of gender-variant children and their parents. Sex Education, V. 13, no 6, p. 644-659.

ROBERTS, A. L., ROSARIO, M., CORLISS, H. L., KOENEN, K. C., \& AUSTIN, S. B. 2012. Elevated risk of posttraumatic stress in sexual minority youths: Mediation by childhood abuse and gender nonconformity. American Journal of Public Health, V. 102, no 8, p. 1587-1593. 
RUBANO, C. 2015. Where do the mermaids stand? Toward a "gender-creative" pastoral sensibility. Pastoral Psychology

RYAN, C., \& RIVERS, I. 2003. Lesbian, gay, bisexual and transgender youth: victimization and its correlates in the USA and UK. Culture, Health E Sexuality, V. 5, no 2, p. 103-119.

SAKETOPOULOU, A. 2014. Mourning the body as bedrock: developmental considerations in treating transsexual patients analytically. Journal of the American Psychoanalytic Association, V. 62, no 5, p. 773-806.

SCHERRER, K. e WOODFORD, M. 2013. Incorporating Content on Gay, Lesbian, Bisexual, Transgender, and Queer Issues in Leading Social Work Journals. Social Work Research, V. 37, no 4, p. 423-431.

SCHNEEBERGERA. R., DIETL, M. F., MUENZENMAIER, K. H., HUBER, C. G. e LANG, U. E. 2014. Stressful childhood experiences and health outcomes in sexual minority populations: a systematic review. Australian and New Zealand Journal of Psychiatry, V. 49, no 9 , p. 1427-45.

SCHWARTZ, D. 2012. Listening to children imagining gender: Observing the inflation of an idea. Journal of Homosexuality, V. 59, no 3, p. 460-479.

SCOTT, J. W. (1989). Gender: A useful category of historical analysis. The American Historical Review, V. 91, nº 5, p. 1053-1075.

SHUMER, D. E., ROBERTS, A. L., REISNER, S. L., LYALL, K., \& AUSTIN, S. B. 2015. Brief report: Autistic traits in mothers and children associated with child's gender nonconformity. Journal of Autism and Developmental Disorders, V. 45, no 5, p. 14891494 .

SILVA, A. L. e OLIVEIRA, A. A. S. 2015. Transexualização em Narrativas de Histórias de Vida sobre a Infância. Estudos e Pesquisas em Psicologia, V. 15, no 2, p. 484-508.

SIMONS, L. K., LEIBOWITZ, S. F. e HIDALGO, M. A. 2014. Understanding gender variance in children and adolescents. Pediatric Annals, V. 43, oㅡ 6.

SLESARANSKY-POE, G,. RUZZI, L., DIMEDIO, C. e STANLEY, J. 2013. Is this the right elementary school for my gender nonconforming child? Journal of LGBT Youth, V. 10, no 1-2, p. 29-44. 
STEENSMA, T. D., VAN DER ENDE, J., VERHULST, F. C. e COHEN-KETTENIS, P. T. 2013. Gender variance in childhood and sexual orientation in adulthood: A prospective study. Journal of Sexual Medicine, V. 10, № 11, p. 2723-2733.

STEIN, E. 2012. Commentary on the treatment of gender variant and gender dysphoric children and adolescents: Common themes and ethical reflections. Journal of Homosexuality, V. 59, no 3, p. 480-500.

STEWART, J. 2009. Commentary on J's story. In: Depalma, R., Atkinson, E. (Ed.). Invisible boundaries: addressing sexualities equalities in children's worlds. Threntham, Inglaterra: Trentham Books.

STIEGLITZ, K. A. 2010. Development, risk, and resilience of transgender youth. Journal of the Association of Nurses in AIDS Care, V. 21, no 3, p. 192-206.

STODDARD, J., LEIBOWITZ, S. F., TON, H. e SNOWDON, S. 2011. Improving medical education about gender-variant youth and transgender adolescents. Child and adolescent psychiatric clinics of North, V. 20, no 4, p. 779-791.

THOMAS, R. N. e BLAKEMORE, J. E. O. 2013. Adults' attitudes about gender nonconformity in childhood. Archives of Sexual Behavior, V. 42, no 3, p. 399-412.

TISHELMAN, A. C., KAUFMAN, R., EDWARDS-LEEPER, L., MANDEL, F. H., SHUMER, D. E. e SPACK, N. P. 2015. Serving transgender youth: Challenges, dilemmas, and clinical examples. Professional Psychology: Research and Practice, V. 46, no 1, p. $37-45$.

TRANSRESPECT VERSUS TRANSPHOBIA. 2016. Trans Murder Monitoring report 2016. TvT Publication Series, V.14

TYLER, T. T. 2015. Our story: The parent and LGBTQ child relational process. Journal of Gay E Lesbian Social Services: The Quarterly Journal of Community E Clinical Practice, V. 27, no1, p. 17-45.

UNIVERSITY OF WASHINGTON, PSYCHOLOGY WRITING CENTER. 2005. Writing a Psychology Literature Review

VANCE, S. R., EHRENSAFT, D. e ROSENTHAL, S. M. 2014. Psychological and medical care of gender nonconforming youth. Pediatrics, V. 134, no 6, p. 1184-92.

VANCE, S.R., HALPERN-FELSHER, B. L. e ROSENTHAL, S. M. 2015. Health care providers' comfort with and barriers to care of transgender youth. Journal of adolescent health, V. 56, no 2, p. 251-253. 
VANDERBURGH, R. 2009. Appropriate therapeutic care for families with prepubescent transgender/gender-dissonant children. Child E Adolescent Social Work Journal, V. 26, no 2, p. 135-154.

WALLACE, R. e RUSSELL, H. 2013. Attachment and shame in gender-nonconforming children and their families: Toward a theoretical framework for evaluating clinical interventions. International Journal of Transgenderism, V 14, no 3, p. 113-126.

WALLIEN, M. S. C. e COHEN-KETTENIS, P. T. 20o8. Psychosexual outcome of gender dysphoric children. Journal of the American Academy of Child E Adolescent Psychiatry, V. 47, p. 1413-1423.

WISEMAN, M. e DAVIDSON, S. 2012 Problems with binary gender discourse: using context to promote flexibility and connection in gender identity. Clinical Child Psychology and Psychiatry, V. 17, no 4, p. 528-537.

WORLD PROFESSIONAL ASSOCIATION FOR TRANSGENDER HEALTH. 2012. Standards ofCareforthe Health ofTranssexual, Transgender, and GenderNonconforming People ( $7^{\mathrm{a}}$ ed.).

WREN, B. 2014. Thinking postmodern and practising in the enlightenment: Managing uncertainty in the treatment of children and adolescents. Feminism \& Psychology, V. 24, no 2, p. 271-291.

ZUCKER, K.J. e BRADLEY, S. J. 1995. Gender identity disorder and psychosexual problems in children and adolescents. New York: Guilford Press.

Recebido em 06/o7/2019.

Aceito em 20/04/2020. 